\title{
Subcutaneously administered methotrexate for rheumatoid arthritis, by prefilled syringes versus prefilled pens: patient preference and comparison of the self-injection experience
}

This article was published in the following Dove Press journal:

Patient Preference and Adherence

6 August 2014

Number of times this article has been viewed

\author{
Winfried Demary' \\ Holger Schwenke ${ }^{2}$ \\ Karin Rockwitz ${ }^{3}$ \\ Peter Kästner ${ }^{4}$ \\ Anke Liebhaber ${ }^{5}$ \\ Ulrich Schoo ${ }^{6}$ \\ Georg Hübner ${ }^{7}$ \\ Uwe Pichlmeier ${ }^{8}$ \\ Cécile Guimbal-Schmolck ${ }^{8}$ \\ Ulf Müller-Ladner ${ }^{9}$ \\ 'Private practice, Hildesheim, \\ Germany; ${ }^{2}$ Private practice, Dresden, \\ Germany; ${ }^{3}$ Private practice, Goslar, \\ Germany; ${ }^{4}$ Outpatient Rheumatism \\ Center, Erfurt, Germany; ${ }^{5}$ Private \\ practice, Halle, Germany; ${ }^{6}$ Private \\ practice, Rheine, Germany; ${ }^{7}$ Private \\ practice, Lingen, Germany; ${ }^{8}$ medac \\ $\mathrm{GmbH}$, Hamburg, Germany; \\ 'Department of Rheumatology and \\ Clinical Immunology, Kerckhoff-Klinik \\ $\mathrm{GmbH}$, Bad Nauheim, Germany
}

Correspondence: Ulf Müller-Ladner Department of Rheumatology and Clinical Immunology, Kerckhoff-Klinik GmbH, Benekestrasse 2-8, D-6123I Bad Nauheim, Germany

$\mathrm{Tel}+4960329962101$

Fax +4960329962104

Email u.mueller-ladner@kerckhoff-klinik.de
Purpose: This multicenter, randomized, crossover study compared preference, ease of use, acceptability, satisfaction, and safety of repeated subcutaneous (SC) self-administrations with prefilled pens and prefilled syringes delivering methotrexate (MTX), in patients with rheumatoid arthritis (RA).

Patients and methods: The study (ClinicalTrials.gov number NCT01793259) enrolled 120 patients requiring initiation or intensification of MTX therapy for RA. Patients were randomized to receive the test drug, a prefilled pen (Metex ${ }^{\circledR} \mathrm{PEN} /$ Metoject ${ }^{\circledR} \mathrm{PEN}$ ), or the reference drug, a prefilled syringe $\left(\right.$ Metex $^{\circledR} /$ Metoject $^{\circledR}$ ), at doses of $15,17.5$, or $20 \mathrm{mg}$ MTX SC once a week for 3 weeks. This was followed by receipt of the reference drug (prefilled syringe) or the test drug (prefilled pen) in a crossover design, with each patient serving as his/her own control. Questionnaires regarding patient preference, the Self-Injection Assessment Questionnaire (SIAQ), and diaries regarding local tolerability were used to document outcomes.

Results: Overall patient preference for the MTX prefilled pen was $75 \%(P<0.0001)$. In a sixitem questionnaire, $73 \%$ to $76 \%$ of the patients preferred the prefilled pen in relation to use, acceptability, and satisfaction, and $67 \%$ of the patients confirmed that it did not take much effort to overcome SC self-injection with the pen. The SIAQ showed no clinical differences, in any domain scores, between both devices. Overall patient attitude towards self-injection at baseline was positive, as was patient experience with both devices during the study. As well, $92 \%$ of physicians and study nurses indicated that they would recommend the MTX prefilled pen to patients for future MTX treatment. The formulations were generally well tolerated.

Conclusion: SC self-injection of MTX with a prefilled pen was generally preferred, by patients with RA, over a prefilled syringe with regard to use, acceptability, and satisfaction. This is supported by the strong appreciation of their attending study nurses and physicians, for its convenience.

Keywords: methotrexate, injection device

\section{Introduction}

Low-dose methotrexate (MTX) has become the disease-modifying antirheumatic drug (DMARD) of choice in the treatment of rheumatoid arthritis (RA). MTX is recommended as the initial choice for the treatment of RA by the European League Against Rheumatism (EULAR) and the American College of Rheumatology (ACR) ${ }^{1-3}$ It is considered the anchor drug by both, on the basis of its efficacy as monotherapy and on the basis of its ability to increase the efficacy of biological DMARDs when used in combination.

MTX is administered weekly at a dose of $7.5 \mathrm{mg}$ to $30 \mathrm{mg}$, either via oral or parenteral route. The parenteral administration shows an improved and more reliable bioavailability compared with the oral formulation, ${ }^{4,5}$ and an improved efficacy, as 
demonstrated in a randomized, double-blind study with 384 patients. ${ }^{6}$ In recent years, the use of subcutaneous (SC) injections gained in importance with the development of prefilled syringes (Metoject ${ }^{\circledR} /$ Metex $^{\circledR}$; medac $\mathrm{GmbH}$, Wedel, Germany) allowing the patient to self-inject at home. Increasing the concentration of the MTX solution to $50 \mathrm{mg} / \mathrm{mL}$ enhanced the comfort, especially at higher doses, by allowing injection of a very small volume. ${ }^{4,7-9}$ The preference of the patient for the $50 \mathrm{mg} / \mathrm{mL}$ prefilled syringe compared with the $10 \mathrm{mg} / \mathrm{mL}$ formulation was shown in a study with 130 RA patients. ${ }^{10}$

A newly developed prefilled pen containing MTX $\left(\right.$ Metex $^{\circledR}$ PEN/Metoject ${ }^{\circledR}$ PEN; medac GmbH) has been recently introduced for greater patient convenience and easier use. In addition, the prefilled pen is provided with a needle-cover system to automatically prevent needlestick injury that could potentially result in secondary infections. This is particularly important when SC injection is not done by the patient himself but by a family member, friend, or health care worker.

This study assessed the experience of patients with RA who used both the prefilled syringes and the prefilled pens, with regard to preference, usability, and tolerability outcomes.

\section{Patients and methods}

\section{Study design}

This was a prospective, multicenter, randomized, open-label, two-period, two-sequence crossover study performed in 12 study centers in Germany. It was designed to assess the number of patients with RA preferring the MTX prefilled pen to the prefilled syringe after 6 weeks of treatment.

\section{Inclusion/exclusion criteria}

Patients were eligible for the study if they were between 18 and 75 years of age, had a diagnosis of RA according to the ACR or ACR/EULAR criteria, ${ }^{1,11}$ and required initiation or intensification of MTX therapy due to RA activity (Disease Activity Score 28 [DAS28] >2.6). ${ }^{12,13}$

Major exclusion criteria for study participation included: prior or other current SC treatment with self-injection; prior or concomitant treatment with biologics; and insufficient knowledge in German to perfectly understand the questionnaires.

The study was performed in accordance with the Good Clinical Practice guidelines recommended by the International Conference on Harmonization of Technical Requirements. ${ }^{14}$ Ethics committees and institutional review boards at the respective study sites approved the trial. Written informed consent was obtained from all patients.

\section{Study intervention}

Patients were randomized to receive the test drug, a prefilled pen $\left(\right.$ Metex $^{\circledR}$ PEN/Metoject ${ }^{\circledR}$ PEN)" or the reference drug, a prefilled syringe $\left(\right.$ Metex $^{\circledR} /$ Metoject $\left.^{\circledR}\right)$, at a dose of $15 \mathrm{mg}$, $17.5 \mathrm{mg}$, or $20 \mathrm{mg}$ MTX SC once a week for 3 weeks. Patients then received the reference drug (prefilled syringe) or the test drug (prefilled pen) in a crossover design, with each patient serving as his/her own control. The SC injections were applied in the abdomen or the upper thigh. Patients were to use a different injection site each time, to minimize any reactions at the injection site. Before the first injection, patients were made familiar with each injection system by the study nurse or the investigator. The first injection was done by the patient himself in the investigator's office under the supervision of the investigator or study nurse. The following two injections were to be performed at home; however, if the patient did not feel comfortable to inject at home, he had the opportunity to perform further injections in the investigator's office. The total treatment duration was 6 weeks, and the MTX dose was to be kept stable during this period.

The study included patients who (1) were MTX-naïve or had already been treated with oral MTX previously, and (2) had no contraindication for MTX at baseline. The patients had to have disease activity at baseline (DAS28 > 2.6) and to require a treatment with MTX, according to their rheumatologist. For these patients, an initiation or an intensification of the MTX treatment was indicated (eg, MTX therapy was switched from oral to parenteral), and the MTX dose was to be increased when necessary. As many rheumatologists in Germany start MTX treatment at a dose of $15 \mathrm{mg}$, the dosage proposed in this study was $15 \mathrm{mg}, 17.5 \mathrm{mg}$, or $20 \mathrm{mg}$ weekly.

Prior and concomitant therapy with other DMARDs, non-steroidal anti-inflammatory drugs, and/or corticosteroids was permitted, as was treatment with oral folic acid once a week. All concomitant medications were to be kept constant during the study.

The following medications were not allowed: prior or combination therapies with a biological DMARD (such as tumor necrosis factor [TNF]- $\alpha$ blockers); drugs causing folate deficiency (eg, sulfonamides and trimethoprim-sulfamethoxazole) (during the study); and live virus vaccinations.

Patients meeting all eligibility criteria were randomized centrally, by the sponsor via fax, to one of the two treatment sequences.

\section{Primary end point}

The primary end point was the number of patients preferring the MTX prefilled pen over the prefilled syringe after 6 weeks of treatment, based on the question "Overall, if you could 
choose, which of both self-injection systems would you prefer for future MTX treatment?".

\section{Secondary end points}

Secondary end points included the following: (1) number of patients preferring the MTX prefilled pen over the prefilled syringe at the end of study, based on a six-item questionnaire related to the overall ease of use, acceptability, and satisfaction; (2) comparison of the self-injection experience of the patients after each treatment period, using the Self-Injection Assessment Questionnaire (SIAQ) version 2.0.15 (3) number of study nurses and investigators preferring the MTX prefilled pen at the end of study; (4) documentation of pen dysfunction, with respect to any information identifying and describing incidents in which the prefilled pen was misused, broken, incapable of being activated, or did not deliver the appropriate dose. Answers were documented by the patient in a diary - at the first and fourth injection, the study nurse/investigator explained and supervised the documentation. In addition, local tolerability and adverse events (AEs) were recorded. Table 1 summarizes the questions and answers concerning preference, usability, and tolerability outcomes.

Table I Preference, usability, and tolerability outcomes

\section{Primary end point - patient preference \\ "Overall, if you could choose, which of both self-injection systems would you prefer for future MTX treatment?" \\ Two categories were suggested: "I prefer the prefilled pen" and "I prefer the prefilled syringe"}

\section{Secondary end points}

Six-item questionnaire related to the overall ease of use, acceptability, and satisfaction with either the MTX prefilled pen or the MTX prefilled syringe to be indicated

"The self-injection is easy to perform using few steps"

"I feel comfortable to perform the self-injection correctly without help"

"The injection system lies comfortably and secure in the hand during the injection"

"I am not scared of being hurt before the injection is done"

"I am not scared of being hurt after the injection is done"

"It does not take much effort to overcome self-injection"

\section{Self-Injection Assessment Questionnaire (SIAQ)}

At baseline, before self-injection:

"In general, how afraid are you of needles?"

"In general, how afraid are you of having an injection?"

"How anxious do you feel about giving yourself an injection?"

"How confident are you about giving yourself an injection in the right way?"

"How confident are you about giving yourself an injection in a clean and sterile way?"

"How confident are you about giving yourself an injection safely?"

Possible answers for all the above items: "not at all"; "a little"; "moderately"; "very"; "extremely"

"Overall, how satisfied are you with your current way of taking your medication?"

Possible answers: "very dissatisfied"; "dissatisfied"; "neither dissatisfied nor satisfied"; "satisfied"; "very satisfied"

After self-injection, at the end of the two periods (the questionnaire covered the following topics, each containing several questions): Feelings about injections

Self-image

Self-confidence

Pain and skin reactions during or after the injection

Ease of use of self-injection device

Satisfaction with self-injection

(Answers were transformed to a score, where a score of one corresponded to the patient's worst experience, and a score of ten corresponded to the patient's best experience)

\section{Study nurse and investigator preference of the MTX prefilled pen at the end of study}

"Overall, how would you rate the experience made by the patients with the prefilled pen during the study?" The answer was given on a five-point semantic Likert-type scale, where a score of one corresponded to the worst experience, and a score of five corresponded to the best experience "Which of both self-injection systems would you recommend to your patients for future MTX treatment?" The following answers could be chosen: "prefilled pen" or "prefilled syringe"

\section{Pen dysfunction}

"Did you notice any defects in the pen, like cracks or breaks?" (Yes/No; if yes, please specify)

"Was it possible to activate the injection by pushing the knob?" (Yes/No, if no, please give details)

"Has the whole solution been injected (the black plunger is located at the bottom of the syringe body)? (Yes/No, if no, please give details) "Did you have any other difficulties using the prefilled pen? (Yes/No; If yes, can you tell me about it?"

Abbreviation: MTX, methotrexate. 


\section{Statistical analyses}

A single-arm chi-square test with a $2.5 \%$ one-sided significance level had a $90 \%$ power to detect an increase in rate of patients preferring the prefilled pen between the null hypothesis rate of $50 \%$ and the alternative rate of $65 \%$ at a sample size of 113 . The sample size was set to 120 patients to adjust for patients excluded from the full analysis.

For statistical analysis of the number of patients preferring the MTX prefilled pen at the end of the study, based on the six-item questionnaire related to the overall ease of use, acceptability, and satisfaction, point estimates of the rates and $95 \%$ confidence intervals were presented. For the statistical analysis of patient preference, a preference for syringe was imputed as the worst-case scenario in cases of missing data.

Exploratory subgroup analyses for age, sex, body mass index (BMI), previous oral MTX treatment, and RA activity was prespecified in the statistical analysis plan. For these analyses, a complete-case analysis without imputation of missing data was performed.

SIAQ scoring was performed in compliance with the user manual. ${ }^{15}$ To account for the crossover design of the study, the individual item score values as well as the domain scores were subjected to analysis of variance (ANOVA). The effects considered in the ANOVA model were: treatment, sequence, and study period, as fixed effects; and patients within sequence, as a random effect. In order to describe any potential difference in domain scores between both devices, the model-based point estimates for the differences and the associated $95 \%$ confidence intervals (CI), using the leastsquares (LS) means and the root of error mean squares from the ANOVA, were presented.

To explore whether there were any relevant sequence or period effects, a logistic regression model was fitted, with sequence and study period as effects. Wilcoxon-tests for matched pairs served as an exploratory tool to assess any potential differences between the local tolerability of the two devices.

The safety population included all patients who received at least one dose of investigational product and for whom at least one postbaseline safety measurement was available. Confirmatory analysis was conducted in the full-analysis set (FAS) including all patients who received at least one injection with each device (syringe and pen) and for whom the primary study parameter was available. For sensitivity analysis, the per-protocol set - although not presented in this paper - was defined to include all patients of the FAS, if all preplanned injections were performed, all major inclusion criteria and none of the major exclusion criteria were fulfilled, and no important protocol violation occurred.
Data were analyzed using the SAS 9.3 software package (SAS Institute Inc., Cary, NC, USA), on the Windows platform.

The study was registered under ClinicalTrials.gov number NCT01793259.

\section{Results}

\section{Analysis of population}

Twelve centers enrolled a total 120 patients into the study. All patients were randomized to receive MTX treatment (in either the sequence pen-syringe or syringe-pen) and were valid for safety analysis. Eight subjects did not complete the study: one patient was lost to follow-up; for another patient, no MTX pen was available at the investigator's site at the time of the study; and six patients discontinued the study due to AEs.

Protocol deviations were recorded for 14 of the 120 patients. These included deviations in the number of planned MTX injections with syringe and pen by 12 patients and deviations in the completion of the six-item questionnaire and overall preference by three patients. In total, 111 patients qualified for the FAS; two patients were excluded due to lacking information for the primary study parameter and seven patients did not receive each type of device at least once.

\section{Demographics and baseline characteristics}

Of the 111 patients (FAS), 81 (73.0\%) were women and 30 $(27.0 \%)$ were men. Mean age was 54.4 years. Mean weight was $80.0 \mathrm{~kg}$, mean height was $168.8 \mathrm{~cm}$, and mean BMI was $28.0 \mathrm{~kg} / \mathrm{m}^{2}$. The mean DAS28 score was 4.5 at baseline, with $88.3 \%$ of the patients having a DAS28 score of $>3.2$. The most common concomitant medical condition was arterial hypertension (36.9\%). A total of $80.2 \%$ of all patients had received oral MTX treatment previously for a mean duration of 31.5 months. In general, baseline characteristics did not differ to a relevant degree between both treatment-sequence groups. Table 2 summarizes demographics and baseline characteristics.

Most patients received an MTX dose of $15 \mathrm{mg}$ at the time of first injection, with both the prefilled pen (73\%) and the prefilled syringe (74\%). Of all patients, $10 \%$ received a dose of $17.5 \mathrm{mg}$, and $14 \%$ received a dose of $20 \mathrm{mg}$. Although patients had the opportunity to return to the investigators' offices for the second and third injections, only one patient $(0.9 \%)$ administered the second/third injection under supervision, and only three patients (2.7\%) administered the fifth/sixth injection under supervision at the investigational site. 
Table 2 Demographics and baseline characteristics

\begin{tabular}{|c|c|c|c|}
\hline Characteristic & $\begin{array}{l}\text { Treatment sequence } \\
\text { pen-syringe }(n=56)\end{array}$ & $\begin{array}{l}\text { Treatment sequence } \\
\text { syringe-pen }(n=55)\end{array}$ & $\begin{array}{l}\text { Total } \\
(n=I I I)\end{array}$ \\
\hline \multicolumn{4}{|l|}{ Sex } \\
\hline Woman, n (\%) & $36(64.3 \%)$ & $45(81.8 \%)$ & $8 \mathrm{I}(73.0 \%)$ \\
\hline Men, n (\%) & $20(35.7 \%)$ & $10(18.2 \%)$ & $30(27.0 \%)$ \\
\hline Age, mean \pm SD (years) & $56.3 \pm 11.0$ & $52.5 \pm 10.2$ & $54.4 \pm 10.8$ \\
\hline Weight, mean \pm SD (kg) & $82.6 \pm 18.2$ & $77.3 \pm 14.6$ & $80.0 \pm 16.7$ \\
\hline Height, mean \pm SD $(\mathrm{cm})$ & $169.0 \pm 9.6$ & $168.7 \pm 8.1$ & $168.8 \pm 8.8$ \\
\hline Body mass index, mean $\pm \mathrm{SD}\left(\mathrm{kg} / \mathrm{m}^{2}\right)$ & $28.8 \pm 5.2$ & $27.2 \pm 4.9$ & $28.0 \pm 5.1$ \\
\hline \multicolumn{4}{|l|}{ Concomitant diseases ( $\geq 10 \%$ of patients) } \\
\hline Arterial hypertension, $\mathrm{n}(\%)$ & $22(39.3 \%)$ & $19(34.5 \%)$ & $4 \mathrm{I}(36.9 \%)$ \\
\hline Osteoarthritis, n (\%) & $8(14.3 \%)$ & $3(5.5 \%)$ & II (9.9\%) \\
\hline Osteoporosis, n (\%) & $6(10.7 \%)$ & $5(9.1 \%)$ & II (9.9\%) \\
\hline Osteopenia, n (\%) & $6(10.7 \%)$ & $4(7.3 \%)$ & $10(9.0 \%)$ \\
\hline Duration of rheumatoid arthritis, mean \pm SD (years) & $3.0 \pm 3.0$ & $3.5 \pm 4.6$ & $3.3 \pm 3.9$ \\
\hline Previous MTX treatment, $\mathrm{n}(\%)$ & $45(80.4 \%)$ & $44(80.0 \%)$ & $89(80.2 \%)$ \\
\hline Duration of previous oral MTX treatment, mean \pm SD (months) & $31.2 \pm 33.2$ & $31.7 \pm 41.0$ & $31.5 \pm 37.1$ \\
\hline $\mathrm{DAS} 28$, mean $\pm \mathrm{SD}$ & $4.5 \pm 1.0$ & $4.5 \pm 1.1$ & $4.5 \pm 1.0$ \\
\hline$>2.6$ to $\leq 3.2$ & $6(10.7 \%)$ & $7(12.7 \%)$ & $13(11.7 \%)$ \\
\hline$>3.2$ to $\leq 5.1$ & $36(64.3 \%)$ & $33(60.0 \%)$ & $69(62.2 \%)$ \\
\hline$>5.1$ & $14(25.0 \%)$ & $15(27.3 \%)$ & $29(26.1 \%)$ \\
\hline
\end{tabular}

Abbreviations: DAS28, Disease Activity Score 28; MTX, methotrexate; SD, standard deviation.

\section{Overall patient preference (primary end point)}

Overall patient preference for the MTX prefilled pen was $75 \%$. Similar statistically significant results were obtained for the complete-case analysis $(\mathrm{n}=109)$, with $76 \%$ for the prefilled pen (95\% CI: 67.0-83.8; $P<0.0001$ ) (Figure 1). The results for the different subgroup stratifications (age, sex, BMI, previous MTX treatment, and baseline DAS28 score) were also similar. In patients reporting to be very or extremely afraid of needles $(n=10)$, the preference for the prefilled pen increased to $90 \%$, while $75 \%$ of the rest of patients preferred the pen (complete-case analysis). Stratified by device sequence, the overall preference of the patient for the prefilled pen was $82 \%$ when MTX was first administered by the prefilled pen and was $67 \%$ when it was first administered by the prefilled syringe.

\section{Six-item questionnaire related to the overall ease of use, acceptability, and satisfaction}

Table 3 summarizes the number of patients with preference for the MTX prefilled pen. In general, between 73\% and $76 \%$ of the patients in both analysis sets preferred the prefilled pen in relation to use, acceptability, and satisfaction. A total $67 \%$ of the patients confirmed that it did not take much effort to overcome self-injection. The respective confidence intervals indicated that the results were statistically significant.

\section{Self-Injection Assessment Questionnaire}

At baseline, patients showed a positive attitude towards self-injection in general. Both device-sequence groups were homogeneously distributed with respect to "PRE" module (completed prior to the first injection) domain results (Table 4).

The experience of the patients with both device systems was positive at the end of the period (Figure 2). In all domains, the mean scores ranged from 7.1 to 9.6. The lowest means were observed for the domain "Self-confidence" ( 7.1 for the pen and 7.5 for the syringe). The highest means were observed for the domain "Pain and skin reactions during or after the injection" (9.6 for the pen versus 9.6 for the syringe).

Thresholds indicating a clinically important difference have not yet been defined and validated for SIAQ domain scores. Assuming a change of at least 0.5 points on a ten-point scale to indicate clinical relevance, no differences were found in any domains scores between both devices.

Post hoc statistical comparisons of the mean scores between both devices revealed for two domains, significant results on an exploratory perspective: for the domain "Selfimage", the score was significantly higher for the pen (LS mean pen 9.14 vs syringe 8.71 [LS mean difference 0.43 ; $P=0.0059])$; in the domain "Self-confidence" the score was significantly higher for the syringe (LS mean pen 7.09 vs syringe 7.53 [LS mean difference $-0.44 ; P=0.0444$ ]).

In summary, domains scores of the SIAQ "POST" module (completed after each sequence period) were not clinically different between pen and syringe. 


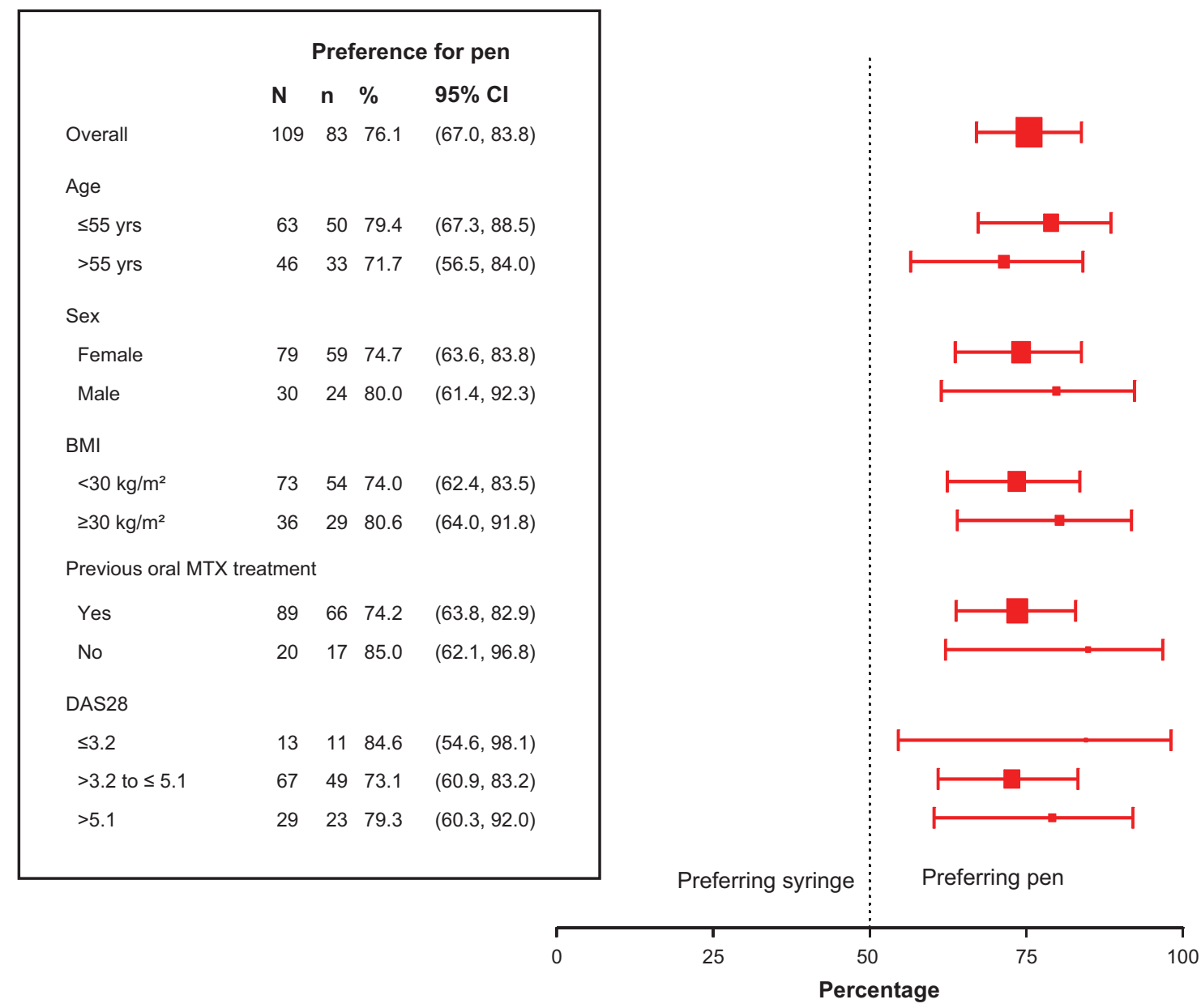

Figure I Overall preference for MTX prefilled pen and stratification by baseline characteristics.

Notes: $\mathrm{N}=$ total number of patients, excluding three patients with missing information $(\mathrm{n}=2)$ and concomitant use of pen and syringe ( $\mathrm{n}=\mathrm{I}$ ) (complete-case analysis); $\mathrm{n}=$ number of patients with preference for pen.

Abbreviations: BMI, body mass index; Cl, confidence interval; DAS28, Disease Activity Score 28; MTX, methotrexate.

Table 3 Preference for MTX prefilled pen applying a six-item questionnaire related to the overall ease of use, acceptability, and satisfaction

\begin{tabular}{|c|c|}
\hline Item & n (\%) \\
\hline \multicolumn{2}{|c|}{ "The self-injection is easy to perform using few steps" } \\
\hline Preference for MTX pen & $81(73.0 \%)$ \\
\hline $95 \% \mathrm{Cl}$ & $63.7-81.0$ \\
\hline \multicolumn{2}{|c|}{ "I feel comfortable to perform the self-injection correctly without help" } \\
\hline Preference for MTX pen & $82(73.9 \%)$ \\
\hline $95 \% \mathrm{Cl}$ & $64.7-81.8$ \\
\hline \multicolumn{2}{|c|}{ "The injection system lies comfortably and secure in the hand during the injection" } \\
\hline Preference for MTX pen & $84(75.7 \%)$ \\
\hline $95 \% \mathrm{Cl}$ & $66.6-83.3$ \\
\hline \multicolumn{2}{|c|}{ "I am not scared of being hurt before the injection is done" } \\
\hline Preference for MTX pen & $82(73.9 \%)$ \\
\hline $95 \% \mathrm{Cl}$ & $64.7-81.8$ \\
\hline \multicolumn{2}{|c|}{ "I am not scared of being hurt after the injection is done" } \\
\hline Preference for MTX pen & $84(75.7 \%)$ \\
\hline $95 \% \mathrm{Cl}$ & $66.6-83.3$ \\
\hline \multicolumn{2}{|c|}{ "It does not take much effort to overcome self-injection" } \\
\hline Preference for MTX pen & $74(66.7 \%)$ \\
\hline $95 \% \mathrm{Cl}$ & $57.1-75.3$ \\
\hline
\end{tabular}

Abbreviations: $\mathrm{Cl}$, confidence interval; MTX, methotrexate. 
Table 4 Frequency distribution of SIAQ "PRE" module items

\begin{tabular}{|c|c|c|c|}
\hline SIAQ item & $\begin{array}{l}\text { Treatment sequence } \\
\text { pen-syringe }(n=56)\end{array}$ & $\begin{array}{l}\text { Treatment sequence } \\
\text { syringe-pen }(n=55)\end{array}$ & $\begin{array}{l}\text { Total } \\
(n=I I I)\end{array}$ \\
\hline \multicolumn{4}{|l|}{ Feeling about injections } \\
\hline \multicolumn{4}{|c|}{ In general, how afraid are you of needles? } \\
\hline Not at all/A little & $44(78.5 \%)$ & 37 (67.3\%) & $81(72.9 \%)$ \\
\hline Moderately & $9(16.1 \%)$ & II (20.0\%) & $20(18.0 \%)$ \\
\hline Very/Extremely & $3(5.4 \%)$ & $7(12.7 \%)$ & $10(9.0 \%)$ \\
\hline \multicolumn{4}{|c|}{ In general, how afraid are you of having an } \\
\hline \multicolumn{4}{|c|}{ injection? } \\
\hline Not at all/A little & $44(78.6 \%)$ & $43(78.2 \%)$ & $87(78.3 \%)$ \\
\hline Moderately & II (19.6\%) & $7(12.7 \%)$ & $18(16.2 \%)$ \\
\hline Very/Extremely & $\mathrm{I}(\mathrm{I} .8 \%)$ & $5(9.1 \%)$ & $6(5.4 \%)$ \\
\hline \multicolumn{4}{|l|}{ How anxious do you feel about giving } \\
\hline \multicolumn{4}{|l|}{ yourself an injection? } \\
\hline Not at all/A little & 39 (69.7\%) & $38(69.1 \%)$ & $77(69.3 \%)$ \\
\hline Moderately & $8(14.3 \%)$ & $7(12.7 \%)$ & $15(13.5 \%)$ \\
\hline Very/Extremely & $9(16.1 \%)$ & $10(18.2 \%)$ & $19(17.1 \%)$ \\
\hline \multicolumn{4}{|c|}{ Self-confidence } \\
\hline \multicolumn{4}{|c|}{ How confident are you about giving yourself } \\
\hline \multicolumn{4}{|c|}{ an injection in the right way? } \\
\hline Extremely/Very & $30(53.5 \%)$ & $32(58.2 \%)$ & $62(55.8 \%)$ \\
\hline Moderately & $12(21.4 \%)$ & II (20.0\%) & $23(20.7 \%)$ \\
\hline A little/Not at all & $13(23.3 \%)$ & $12(21.9 \%)$ & $25(22.5 \%)$ \\
\hline Missing data & $\mathrm{I}(\mathrm{I} .8 \%)$ & $0(0.0 \%)$ & $\mathrm{I}(0.9 \%)$ \\
\hline \multicolumn{4}{|c|}{ How confident are you about giving yourself } \\
\hline \multicolumn{4}{|c|}{ an injection in a clean and sterile way? } \\
\hline Extremely/Very & $35(62.5 \%)$ & 40 (72.7\%) & $75(67.6 \%)$ \\
\hline Moderately & $10(17.9 \%)$ & $6(10.9 \%)$ & $16(14.4 \%)$ \\
\hline A little/Not at all & $10(17.9 \%)$ & $9(16.3 \%)$ & $19(17.1 \%)$ \\
\hline Missing data & $\mathrm{I}(\mathrm{I} .8 \%)$ & $0(0.0 \%)$ & I (0.9\%) \\
\hline \multicolumn{4}{|c|}{ How confident are you about giving yourself } \\
\hline \multicolumn{4}{|c|}{ an injection safely? } \\
\hline Extremely/Very & $33(58.9 \%)$ & $35(63.6 \%)$ & $68(6 \mid .3 \%)$ \\
\hline Moderately & $12(21.4 \%)$ & II (20.0\%) & $23(20.7 \%)$ \\
\hline A little/Not at all & $10(17.9 \%)$ & $9(16.4 \%)$ & $19(17.1 \%)$ \\
\hline Missing data & $\mathrm{I}(\mathrm{I} .8 \%)$ & $0(0.0 \%)$ & I (0.9\%) \\
\hline \multicolumn{4}{|c|}{ Satisfaction with self-injection } \\
\hline \multicolumn{4}{|c|}{ Overall, how satisfied are you with your } \\
\hline \multicolumn{4}{|c|}{ current way of taking your medication? } \\
\hline Very satisfied/Satisfied & $30(53.6 \%)$ & 30 (54.5\%) & $60(54.0 \%)$ \\
\hline Neither dissatisfied nor satisfied & $14(25.0 \%)$ & 14 (25.5\%) & $28(25.2 \%)$ \\
\hline Dissatisfied/Very dissatisfied & $8(14.2 \%)$ & $7(12.8 \%)$ & $15(13.5 \%)$ \\
\hline Missing data & $4(7.1 \%)$ & $4(7.3 \%)$ & $8(7.2 \%)$ \\
\hline
\end{tabular}

Abbreviations: SIAQ, Self-Injection Assessment Questionnaire; PRE module, completed prior to the first injection.

\section{Study nurse and investigator preference}

A total of six study nurses and six investigators completed the questionnaire on the overall experience with the MTX prefilled pen and syringe, of whom eleven $(92 \%)$ gave the MTX prefilled pen the two highest of five possible rankings and indicated that they would recommend the MTX prefilled pen to patients for future MTX treatment.

\section{Pen dysfunction}

In all, 18 patients documented instances of dysfunction or misuse of the MTX prefilled pen or other difficulties in their diary, which were grouped into four categories:
"Could not inject the entire solution" ( $\mathrm{n}=9$ ); "Could not activate the injection" $(\mathrm{n}=7)$; "Any defects in the pen, like cracks and breaks" $(n=3)$; or "Other difficulties" $(n=9)$. As data clarification of diary entries was not possible, some inconsistencies and redundancies could not be avoided.

Nine patients reported that the solution could not be injected completely, for ten separate injections (3\% of all injections with the pen). Of these, two patients stated that the solution was only partly injected because they did not wait long enough to complete the injection. Two patients reported that at least one drop of the medication remained on the skin after the injection. 


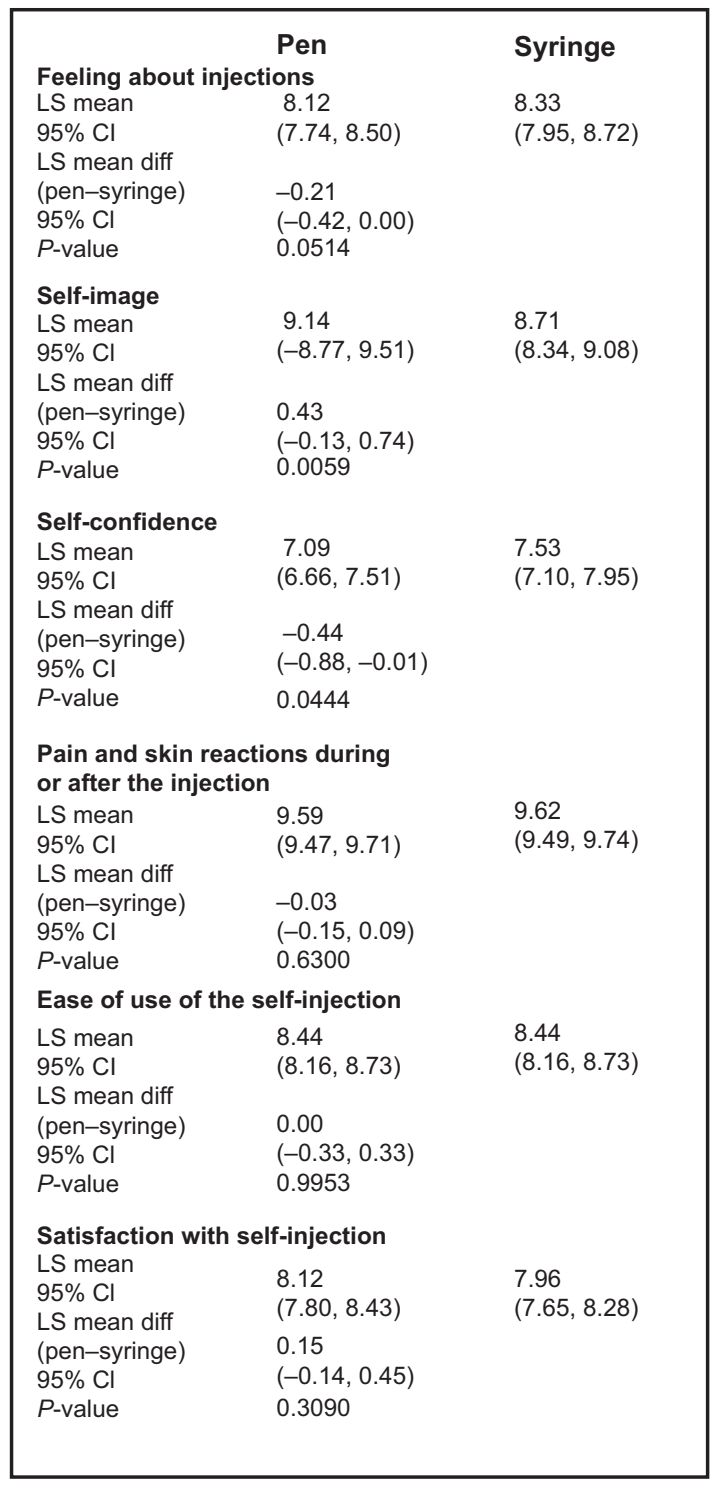

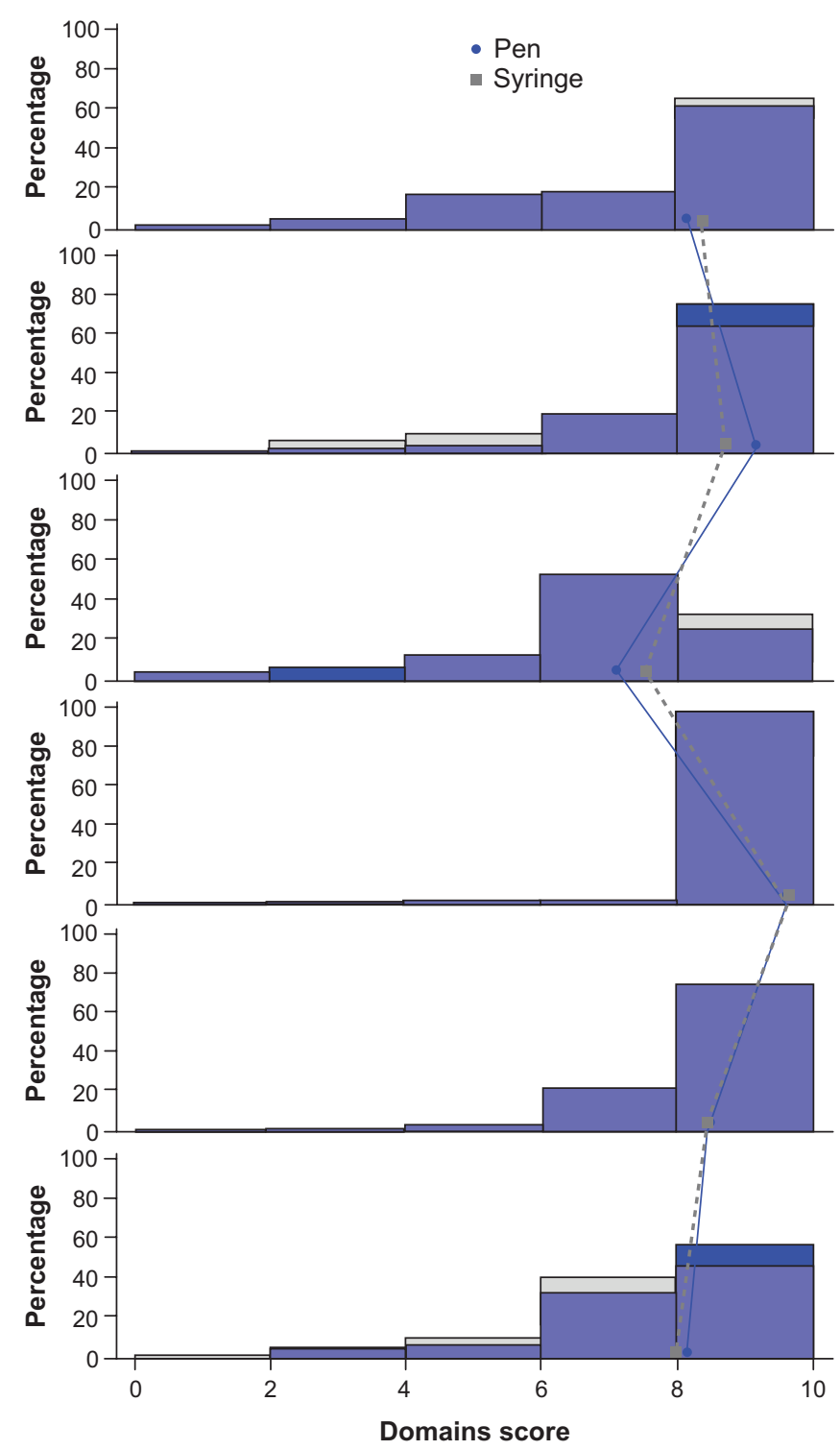

Figure 2 Distribution of domain scores of the SIAQ POST-module (histogram for each device; LS means among figures are connected). Abbreviations: $\mathrm{Cl}$, confidence interval; diff, difference; LS, least square; SIAQ, Self-Injection Assessment Questionnaire.

Of the seven patients who reported their difficulty with or inability to activate the injection (by pushing the knob), for seven separate injections ( $2 \%$ of all injections with the pen), four patients imputed the problem to the pen; two patients reported difficulties with pen activation, and one patient reported incorrect pen use.

Three patients reported "defects in the pen", for three separate injections ( $1 \%$ of all injections with the pen). However, these defects were unrelated to cracks or breaks but rather, to the inability of the patients to activate the injection.

Other difficulties using the prefilled pen were reported by nine patients, for 16 separate injections ( $5 \%$ of all injections with the pen); these included difficulties during activation $(n=4)$, difficulties with cap removal $(n=1)$, and reasons unlikely to be related to device dysfunction $(n=4)$.

\section{Local tolerability}

Table 5 presents the pooled analysis showing the cumulative frequencies of patients with symptoms at the injection sites, as rated by the patient or the investigator within 48 hours after injection.

In all, 52 patients (44.8\%) using the pen and 54 patients $(46.2 \%)$ using the syringe experienced local symptoms, as rated by the patient or the investigator. Most symptoms were mild in intensity. Patients using the pen experienced hematoma most often (33 patients [28.4\%]), followed by pain (25 patients [21.6\%]), redness (19 patients [16.4\%]), itching (ten patients [8.6\%]), and swelling (eight patients [6.9\%]). Patients using the syringe experienced redness most often (36 patients [30.8\%]), followed by hematoma (26 patients [22.2\%]), pain and 
Table 5 Frequency of patients with symptoms at the injection sites, as rated by patient or the investigator, within 48 hours after injection (safety analysis set; $\mathrm{n}=120$ )

\begin{tabular}{lll}
\hline Local tolerability symptoms & Pen & Syringe \\
\hline Number of patients with available data & $116(100.0 \%)$ & $117(100.0 \%)$ \\
Patients with any event & $52(44.8 \%)$ & $54(46.2 \%)$ \\
Pain & & \\
Any event & $25(21.6 \%)$ & $10(8.5 \%)$ \\
Mild & $23(19.8 \%)$ & $8(6.8 \%)$ \\
$\quad$ Moderate & $2(1.7 \%)$ & $2(1.7 \%)$ \\
Redness & & \\
Any event & $19(16.4 \%)$ & $36(30.8 \%)$ \\
Mild & $14(12.1 \%)$ & $32(27.4 \%)$ \\
Moderate & $5(4.3 \%)$ & $4(3.4 \%)$ \\
Swelling & & \\
Any event & $8(6.9 \%)$ & $9(7.7 \%)$ \\
Mild & $7(6.0 \%)$ & $7(6.0 \%)$ \\
Moderate & $1(0.9 \%)$ & $2(1.7 \%)$ \\
Hematoma & & \\
Any event & $33(28.4 \%)$ & $26(22.2 \%)$ \\
Mild & $24(20.7 \%)$ & $23(19.7 \%)$ \\
Moderate & $8(6.9 \%)$ & $3(2.6 \%)$ \\
Severe & $1(0.9 \%)$ & $0(0.0 \%)$ \\
Itching & & $10(8.5 \%)$ \\
Any event & $10(8.6 \%)$ & $8(6.8 \%)$ \\
Mild & $7(6.0 \%)$ & $1(0.9 \%)$ \\
Moderate & $2(1.7 \%)$ & $1(0.9 \%)$ \\
Severe & $1(0.9 \%)$ & 0.9648 \\
\hline Notes & & 0.1583 \\
\end{tabular}

Notes: aP-value for testing difference between devices (Wilcoxon test for matched pairs).

itching (ten patients [8.5\%] for each), and swelling (nine patients [7.7\%]).

Differences in local tolerability between both devices were found for pain and redness. Significantly more patients experienced pain when using the pen compared with the syringe $(P=0.0019)$. Significantly more patient experienced redness when using the syringe compared with the pen $(P=0.0421)$.

\section{Safety}

The safety data are based on all 120 patients who were eligible for safety analysis. Both MTX pen and syringe treatments were generally well tolerated. Table 6 summarizes the AE information.

In all, 38 patients (31.7\%) reported AEs. The most frequent AEs were related to infections and infestations (18 patients [15.0\%]), with nasopharyngitis being most frequent (12 patients [10.0\%]); gastrointestinal disorders (13 patients [10.8\%]), with nausea being most common (nine patients [7.5\%]); nervous system disorders (five patients [4.2\%]); and skin and subcutaneous tissue disorders (five patients $[4.2 \%])$. AEs related to general disorders and administration site conditions were reported by four patients (3.3\%), investigations by three patients $(2.5 \%)$, and psychiatric disorders by two patients $(1.7 \%)$. AEs related to other organ system classes were reported by only one patient each.
Seven patients $(6 \%)$ discontinued MTX treatment due to AEs, of which nausea was the most common (five patients [4\%]). Five patients (4\%) interrupted MTX treatment; the most common reason was increased hepatic enzymes, which occurred in two patients (2\%). Hepatic enzyme increases included elevated gamma-glutamyl transpeptidase (GGT) (129 U/L) in one patient and elevated aspartate aminotransferase (AST) (207 U/L) and alanine aminotransferase (ALT) (306 U/L) in another patient; all were reported at the time of the fourth injection. No relevant differences between the two devices were present with regard to number and type of AEs. None of the AEs was considered serious.

\section{Discussion}

In this randomized, open-label, crossover study, $76 \%$ of all patients with RA expressed their overall preference with the MTX prefilled pen over the prefilled syringe, the result being highly statistically significant $(P, 0.0001)$. Subgroup stratifications for age, sex, BMI, previous MTX treatment, and baseline DAS28 score yielded similar results. For all subgroups, the preference for the MTX prefilled pen ranged from $73 \%$ to $85 \%$, and the lower limits of the respective $95 \%$ CIs were well above $50 \%$.

This preference for the prefilled pen was further supported by the results of the six-item questionnaire assessing use, 
Table 6 Frequency of patients with adverse events (safety analysis set; $n=\mid 20$ )

\begin{tabular}{|c|c|c|c|}
\hline MedDRA system organ class & Pen & Syringe & Total \\
\hline Preferred term & n (\%) & n (\%) & n (\%) \\
\hline Number of patients & $116(100.0 \%)$ & $117(100.0 \%)$ & $120(100.0 \%)$ \\
\hline Patients with any event & $24(20.7 \%)$ & $19(16.2 \%)$ & $38(31.7 \%)$ \\
\hline Infections and infestations (any event) & $10(8.6 \%)$ & $8(6.8 \%)$ & $18(15.0 \%)$ \\
\hline Nasopharyngitis & $7(6.0 \%)$ & $5(4.3 \%)$ & $12(10.0 \%)$ \\
\hline Cystitis & I (0.9\%) & I (0.9\%) & $2(1.7 \%)$ \\
\hline Gastrointestinal disorders (any event) & $10(8.6 \%)$ & $3(2.6 \%)$ & $13(10.8 \%)$ \\
\hline Nausea & $6(5.2 \%)$ & $3(2.6 \%)$ & $9(7.5 \%)$ \\
\hline Vomiting & $2(1.7 \%)$ & $0(0.0 \%)$ & $2(1.7 \%)$ \\
\hline Nervous system disorders (any event) & $4(3.4 \%)$ & I $(0.9 \%)$ & $5(4.2 \%)$ \\
\hline Headache & $3(2.6 \%)$ & $0(0.0 \%)$ & $3(2.5 \%)$ \\
\hline Dizziness & I (0.9\%) & I $(0.9 \%)$ & $2(1.7 \%)$ \\
\hline Skin and subcutaneous tissue disorders (any event) & I $(0.9 \%)$ & $4(3.4 \%)$ & $5(4.2 \%)$ \\
\hline General disorders and administration site conditions (any event) & I $(0.9 \%)$ & $3(2.6 \%)$ & $4(3.3 \%)$ \\
\hline Investigations (any event) & $2(1.7 \%)$ & I $(0.9 \%)$ & $3(2.5 \%)$ \\
\hline Hepatic enzymes increased & $2(1.7 \%)$ & $0(0.0 \%)$ & $2(1.7 \%)$ \\
\hline Psychiatric disorders (any event) & I (0.9\%) & I (0.9\%) & $2(1.7 \%)$ \\
\hline
\end{tabular}

Abbreviation: MedDRA, Medical Dictionary for Regulatory Activities.

acceptability, and satisfaction. For all but one item, patient preference for the prefilled pen ranged from $73 \%$ to $76 \%$. About two-thirds (67\%) of the patients confirmed that the use of the prefilled pen did not take much effort to overcome selfinjection.

The positive patient perception of the MTX prefilled pen was also confirmed by the nurses and investigators involved in this study, who reported "positive" to "best" experience for all patients at their site with the prefilled pen. All but one site would recommend the prefilled pen for the future treatment of their patients. However, due to the small number of participating investigators or nurses $(n=12)$, these results should be interpreted with caution.

Results from the SIAQ showed no clinically relevant differences between both devices in all domain scores of the SIAQ POST module. Generally, overall patient attitude towards self-injection at baseline was positive, as was patient experience with both devices during the study. However, testing differences in mean scores between both devices revealed two domains with significantly different scores: the score for the domain "self-image" was significantly higher for the pen and the score for the domain "self-confidence" was significantly higher for the syringe.

Whereas questionnaire outcomes concerning overall preference, use, acceptability, and satisfaction clearly favored the use of the prefilled pen, the SIAQ POST module domain and item scores showed no patient preference towards either of the two devices.

Regarding device dysfunction and misuse, as reported in patient diaries, nine patients were unable to inject the whole solution volume, seven were unable to activate the injection device, three reported a defect, and nine reported other difficulties. In relation to the total number of used MTX prefilled pens $(n=347)$, the whole solution was not injectable in $3 \%$, the injection device was not activated in $2 \%$, a defect was reported in $1 \%$, and other difficulties were reported in $5 \%$. As clarification of data in the diary entries was not possible, some inconsistencies and redundancies could not be avoided; among these, a review of individual comments revealed that for six patients, one injection per patient was not performed with the initial pen, which had to be replaced. All other patient reports were related to observations, incorrect use of the pen, or difficulties in pen use.

These reported difficulties or inability to use the pen may be imputed to insufficient training at the physicians' offices, to limitation in the use of the pen by the patients, or to penrelated technical problems.

Three of the six pens that required replacement were from a single investigator site and the need for replacement may have been due to insufficient device training at this site. Moreover, looking at the inability to inject the entire solution volume revealed an increased occurrence at the second injection in comparison with the third injection, for the prefilled pens. This time point corresponds to the first injection by the patient at home, without any supervision by a health care professional, suggesting the number of problems encountered by the patients may decrease with the number of injections performed. As the patients reported difficulties or inability to use the pen while they were at home, the issues were not evaluated by a health care professional and therefore cannot definitely be clarified here.

With regard to local tolerability at the injection site, significantly more patients experienced pain when using the 
pen compared with the syringe ( $P=0.0019)$, and significantly more patients experienced redness when using the syringe compared with the pen $(P=0.0421)$. Both formulation devices were generally well tolerated.

Often, patients associate SC injections with pain, discomfort, blood, and cross infection. Compared with the syringe system, the prefilled pen enables a process, where - for self-injection the patient simply presses a button and is therefore not directly involved with the needle, resulting in minimal anxiety and perception of pain. In addition, the needle cover minimizes the risk of needlestick injury. These advantages and the favorable patient, physician, and nurse assessments support the use of the prefilled pen for MTX administration in patients with RA.

\section{Conclusion}

SC self-injection of MTX with a prefilled pen was generally preferred over a prefilled syringe with regard to use, acceptability, and satisfaction, by patients with RA. This is supported by the strong appreciation of its convenience, by their attending study nurses and physicians.

\section{Acknowledgments}

The authors wish to thank all patients and nursing staff involved in the study, as well as the following rheumatologists: M Bohl-Bühler, Potsdam; A Gause, Hamburg; B Heilig, Heidelberg; and P Wagener; Bruchhausen-Vilsen.

Martin Bornemann provided medical writing support on behalf of medac GmbH, Germany. The SIAQ questionnaire was used with the permission of the copyright holder, UCB Pharma, S.A., Belgium.

\section{Disclosure}

C Guimbal-Schmolck and U Pichlmeier are employees of medac $\mathrm{GmbH}$. The other authors were investigators in the medac study. The authors report no other conflicts of interest in this work.

\section{References}

1. Smolen JS, Landewé R, Breedveld FC, et al. EULAR recommendations for the management of rheumatoid arthritis with synthetic and biological disease-modifying antirheumatic drugs. Ann Rheum Dis. 2010;69(6): 964-975.
2. Singh JA, Furst DE, Bharat A, et al. 2012 update of the 2008 American College of Rheumatology recommendations for the use of disease-modifying antirheumatic drugs and biologic agents in the treatment of rheumatoid arthritis. Arthritis Care Res (Hoboken). 2012;64(5):625-639.

3. Saag KG, Teng GG, Patkar NM, et al; American College of Rheumatology. American College of Rheumatology 2008 recommendations for the use of nonbiologic and biologic disease-modifying antirheumatic drugs in rheumatoid arthritis. Arthritis Rheum. 2008;59(6):762-784.

4. Hoekstra M, Haagsma C, Neef C, Proost J, Knuif A, van de Laar M. Bioavailability of higher dose methotrexate comparing oral and subcutaneous administration in patients with rheumatoid arthritis. J Rheumatol. 2004;31(4):645-648.

5. Kubisch SA, Weigand T, Plischke H, Menninger H. Bioverfugbarkeit von Methotrexat (MTX) in unterschiedlichen Applikationsarten [Bioavailability of Methotrexate (MTX) following different routes of application]. Akt Rheumatol. 2004;29:197-200. German.

6. Braun J, Kästner P, Flaxenberg P, et al; MC-MTX.6/RH Study Group. Comparison of the clinical efficacy and safety of subcutaneous versus oral administration of methotrexate in patients with active rheumatoid arthritis: results of a six-month, multicenter, randomized, double-blind, controlled, phase IV trial. Arthritis Rheum. 2008;58(1):73-81.

7. Auvinet B, Jarrier I, Le Levier F, Pegon Y, Turcant A, Allain P. [Compared bioavailability of methotrexate administered orally or intramuscularly in rheumatoid arthritis]. Presse Med. ;21(17):822. French.

8. Cronstein BN. Low-dose methotrexate: a mainstay in the treatment of rheumatoid arthritis. Pharmacol Rev. 2005;57(2):163-172.

9. Herman RA, Veng-Pedersen P, Hoffman J, Koehnke R, Furst DE. Pharmacokinetics of low-dose methotrexate in rheumatoid arthritis patients. J Pharm Sci. 1989;78(2):165-171.

10. Müller-Ladner U, Rockwitz K, Brandt-Jürgens J, et al; MC-MTX.10/RH Study Group. Tolerability and patient/physician satisfaction with subcutaneously administered methotrexate provided in two formulations of different drug concentrations in patients with rheumatoid arthritis. Open Rheumatol J. 2010;4:15-22.

11. Arnett FC, Edworthy SM, Bloch DA, et al. The American Rheumatism Association 1987 revised criteria for the classification of rheumatoid arthritis. Arthritis Rheum. 1988;31:315-324.

12. Prevoo MLL, van 't Hof MA, Kuper HH, van Leeuwen MA, van de Putte LB, van Riel PL. Modified disease activity scores that include twenty-eight-joint counts: development and validation in a prospective longitudinal study of patients with rheumatoid arthritis. Arthritis Rheum. 1995;38(1):44-48.

13. van Riel PL, Schumacher HR. How does one assess early rheumatoid arthritis in daily clinical practice? Best Pract Res Clin Rheumatol. 2001; 15(1):67-76.

14. ICH harmonisation for better health [webpage on the Internet]. Geneva: Harmonised Tripartite Guideline for Good Clinical Practice (E6). Available from: http://www.ich.org/products/guidelines/efficacy/efficacysingle/article/good-clinical-practice.html. Accessed May 30, 2014.

15. Keininger D, Coteur G. Assessment of self-injection experience in patients with rheumatoid arthritis: psychometric validation of the Self-Injection Assessment Questionnaire (SIAQ). Health Qual Life Outcomes. 2011;9:2.
Patient Preference and Adherence

\section{Publish your work in this journal}

Patient Preference and Adherence is an international, peer-reviewed, open access journal that focuses on the growing importance of patient preference and adherence throughout the therapeutic continuum. Patient satisfaction, acceptability, quality of life, compliance, persistence and their role in developing new therapeutic modalities and compounds to optimize

\section{Dovepress}

clinical outcomes for existing disease states are major areas of interest for the journal. This journal has been accepted for indexing on PubMed Central. The manuscript management system is completely online and includes a very quick and fair peer-review system, which is all easy to use. Visit http://www. dovepress.com/testimonials.php to read real quotes from published authors. 